\title{
Performance Analysis According to RSU Range of VANET-based Communication Vehicle
}

\author{
JinYeong Um \\ Dept. of Computer Science \& Engineering, Dongguk Univ. 30, Pildong-ro 1-gil, \\ Jung-gu, Seoul, Korea \\ mog07@dongguk.edu
}

\begin{abstract}
VANET is a special kind of MANET adapted to the communication between vehicles. It has the characteristics of moving at high speed, and is classified into V2I and V2V communication methods. In order to develop VANET-based vehicle wireless communication technology, it is necessary to establish a test environment and synchronize vehicle mobility in various road scenarios. However, when the experimental environment is built, the equipment is expensive, and the road traffic situation is difficult to predict. The purpose of this paper is to implement a simulation of a Vehicluar Ad-Hoc network for urban environment that can be used for testing purposes. Such environment can be used to design better MAC protocols in VANET. In this paper, we propose a scheme for experiments in a similar environment to actual urban traffic, and analyze the performance by dividing it into V2I and V2V nodes according to the RSU coverage.
\end{abstract}

Keywords: Wave, VANET, V2I, V2V, RSU

\section{Introduction}

With the increase of vehicles and the development of technology, the damage of lives and property due to safety problems such as traffic accidents is increasing greatly. In order to solve this problem, various technologies have been researched and developed. Among them, many researches on safety systems through WAVE (wireless access in vehicular environments) communication have been conducted. V2X (Vehicle to Everything) is a communication technology that uses wired / wireless networks to exchange information between vehicles and infrastructure. It includes all forms of communication technology between vehicles and various devices on the road. That is, V2X includes Vehicle to Vehicle (V2V), Vehicle to road Infrastructure (V2I, Vehicle to Infra), and Vehicle to Pedestrian (V2P). As vehicle communication may cause mobility problems due to high speed driving, development of vehicle wireless communication technology based on VANET (Vehicular Ad-hoc Network) in consideration of mobility is being actively performed [1].

In order to develop wireless communication technology with VANET-based vehicles, when the proposed communication technique is tested using a real vehicle, the difference in performance must be tested by a real vehicle. In addition, testbeds of different scenarios must be built on real roads and vehicle mobility synchronized to the scenario. However, it is difficult to experiment according to the scenario because the equipment is expensive and the road traffic

Article history:

Received (January 10, 2020), Review Result (February 14, 2020), Accepted (March 22, 2020) 
situation isn't expected [2]. And because the experiment with the vehicle may cause a safety problem.

In this paper, we perform a virtual experiment using a network simulator before conducting a large-scale or actual test, and use the data obtained to predict the actual test result and present a method to increase the effectiveness of the experiment. The schemes for building a virtual experiment environment using network simulators were presented. However, in the related studies [2], it is difficult to analyze the performance of each communication by analyzing the performance of all nodes without considering V2V and V2I communication. In this paper, we construct a road-like environment by combining the road traffic simulator SUMO, which supports the traffic-like environment of the real city, and the Veins framework for VANET wireless communication experiments. In this environment, we present a method for analyzing IEEE 802.16 WAVE and performance by classifying V2I and V2V nodes according to RSU (Road Side Units) reception range, rather than performance analysis for all nodes.

\section{VANET simulator}

AS shown in [Figure 1], VANET communication is divided into V2V and V2I. V2V refers to vehicle to vehicle communications and is based on IEEE 802.11p and WAVE communications. Each vehicle detects surrounding hazards by exchanging its own vehicle information such as speed and GPS (Global Positioning System), and surrounding situation information through perception of surrounding conditions while driving. V2I refers to the communication between the WAVE communication terminal of a vehicle and neighboring base stations. It is a communication method for providing signal information and safety services at intersections, priority signal control for emergency vehicles, and collecting and providing traffic information [3].

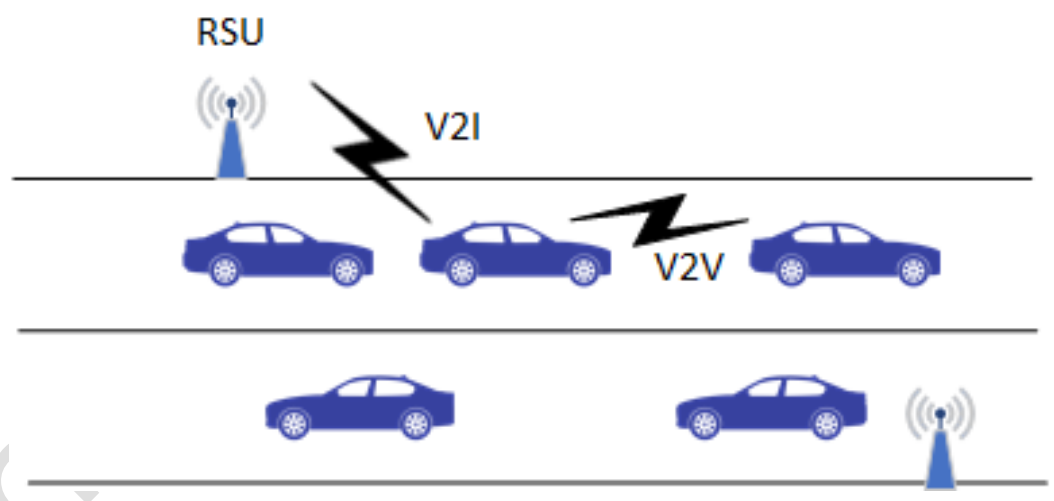

Figure 1. VANET Communication configuration

[Figure 2] shows the open source framework which performs VANET simulation by connecting network simulator OMNeT ++ and road traffic simulation [4]. OMNET ++ is an object-oriented event-based simulator engine and an open source simulation program that can be used for various types of simulations, including communication protocols, computer networks, multiprocessors, and distributed systems. There is a lack of connectivity and mobility to simulate a fast-moving vehicle network [5][6][7]. MiXiM(Mixed Simulator) is an OMNeT++ modeling framework created for mobile and fixed wireless networks (wireless sensor networks, body area networks, ad-hoc networks, vehicular networks, etc.). It offers detailed models of radio wave propagation, interference estimation, radio transceiver power consumption and wireless MAC protocols [6][8]. 
SUMO is a free and open traffic simulation suite. it allows modeling of intermodal traffic systems including road vehicles, public transport and pedestrians. SUMO is also included with various supporting tools that can handle different task, such as route finding, visualization, network import and emission calculation. It can further be enhanced with custom models and provides various features to remotely control the simulation SUMO has been widely used to tackle with variety of research project, for example evaluating modern algorithm in increasing the performance of traffic lights [9].

Veins were developed based on MiXiM. It runs two simulators in parallel over SUMO and OMNeT ++ via TCP sockets, and receives information about vehicle generation and mobility from SUMO, allowing each vehicle to be recognized as a node in OMNeT ++ to enable VANET communication simulation. Supporting road traffic, the SUMO simulator supports road networks including roads, streets, traffic lights, intersections, and details such as car generation, speed and direction of each car. In addition, starting point and destination, time and location can be displayed, and maps can be extracted from OpenStreetMap to provide an environment similar to road traffic in actual cities [2][10][11].

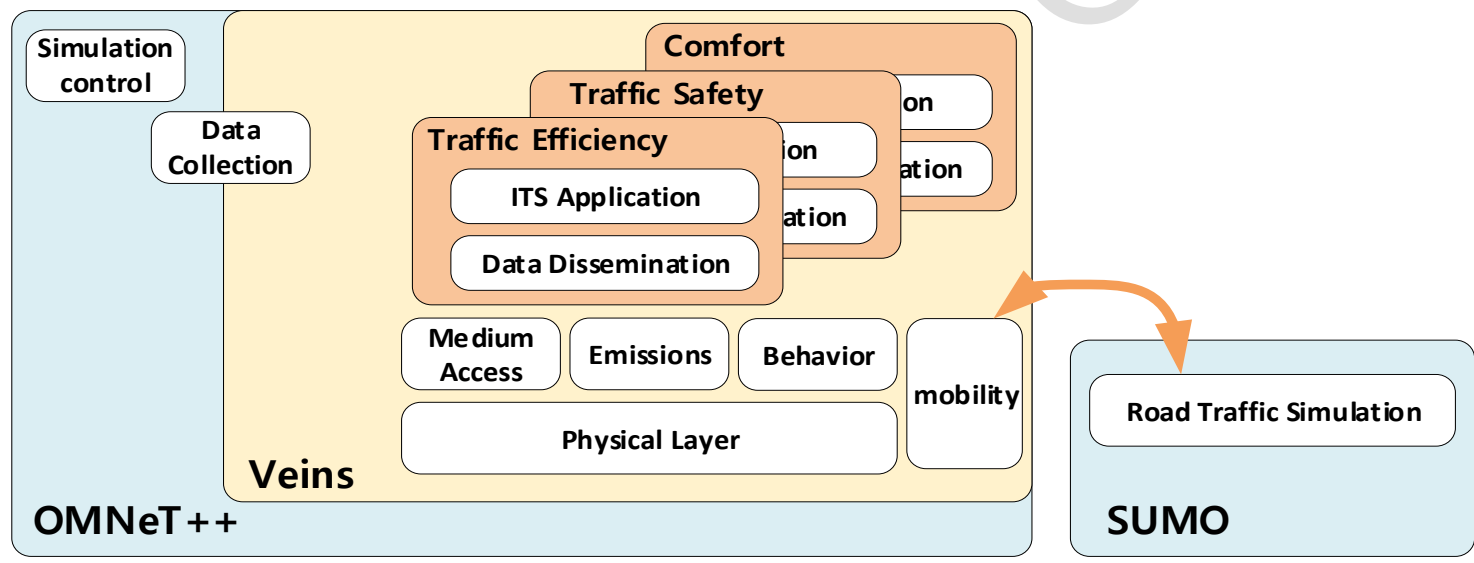

Figure 2. Framework of OMNet++, Veins and SUMO

\section{VANET experiment environment for RSU receiving range control}

In the previous study [2], a study was conducted on vehicle traffic for 24 hours in $400 \mathrm{~km} 2$ of K"oln, in Germany. However, without considering V2V and V2I communication separately, there is disadvantage that performance analysis for each communication can't be performed by analyzing the performance of all communication nodes. It isn't possible to perform performance analysis when developing a communication protocol of a vehicle network in which heterogeneous networks are mixed.

In this paper, we consider a simple topology as shown in [Figure 3] to classify V2V and V2I. All communicating nodes are divided into I-RNR (Vehicles within RSU's communication range) and Non-RNR (Vehicles outside the RSU's range of communication) located on the road side, and the number of successful beacons of I-RNR and Non-RNR caculated. When IRNR, it means V2I, and when Non-RNR, it means V2V. 


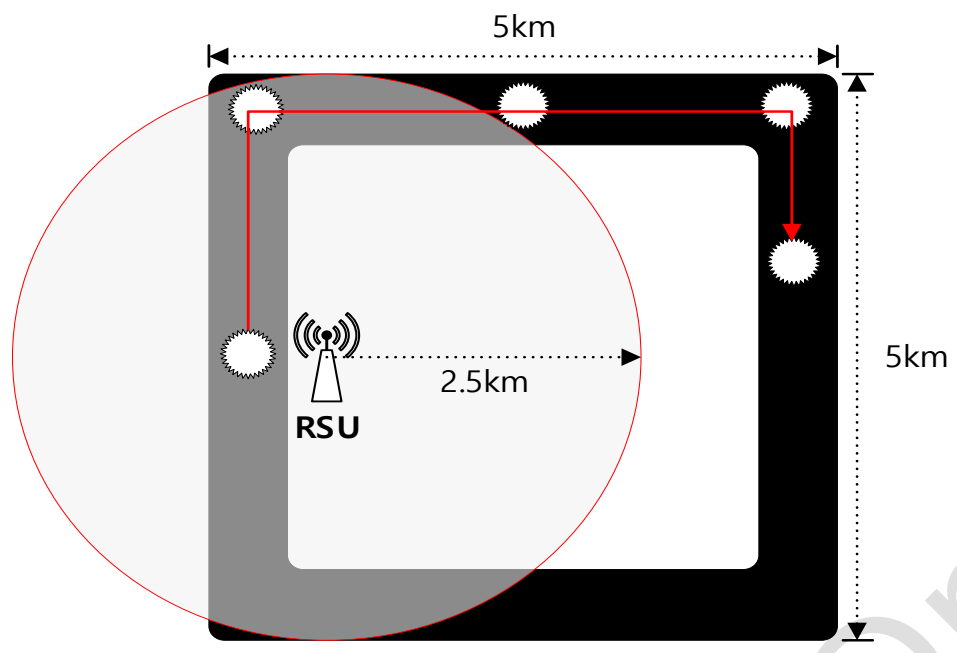

Figure 3. I-RNR and Non-RNR simple topology

Veins communicate based on vehicle node information received from SUMO through TCP socket. The operation process of each node is as follows.

1) Each node including RSU performs channel switching

2) If a Beacon event occurs during channel switching, broadcast a Beacon message to nodes within the Beacon range.

3) If the transmitting node is within the receiving range of the RSU, it transmits the packet to the destination node including the RSU and all the nodes, otherwise it communicates between vehicles (V2V) and transmits the packet to all nodes of the receiving range except the RSU.

4) If the packet transmission is completed and the simulation end time, the process ends. If the simulation end time is not, proceed to step 1) again.

\section{Performance analysis}

Experiment with the scenario described in section 3. Enter the I-RNR communication range (or non-RNR communication range) for the entire experiment time. After selecting one node among all nodes, calculate the number of beacon transmissions for all adjacent nodes. The beacon transmission probability during the experiment time is calculated using the number of beacon transmissions of all nodes and the number of beacons transmitted by the selected node. A road topology consisting of SUMO is used as shown in [Figure 3], and the speed of each vehicle node is limited to $15-70 \mathrm{~km}$. Vehicle nodes are produced every second. Over time, the number of neighboring nodes is increased to increase the total number of beacon transmissions, thereby increasing the probability of collision.

[Table 1] shows the beacon size, the bacon transmission bandwidth, the minimum signal attenuation threshold, and the parameter values of channel bit rate (CBR) and maximum transmission power.

Table 1. Experimental parameter

\begin{tabular}{|c|c|}
\hline Parameter & value \\
\hline Beaconing rate & $10 \mathrm{~Hz}$ \\
\hline
\end{tabular}




\begin{tabular}{|c|c|}
\hline Beacon size & $256 \mathrm{bytes}$ \\
\hline Minimum signal attenuation threshold & $-89 \mathrm{dBm}$ \\
\hline Maximum transmission power & $20 \mathrm{~mW}$ \\
\hline
\end{tabular}

Since the coordinator exists when the RSU is within the reception range, it was expected that the beacon transmission probability is higher than that when the RSU is not within the reception range. However, as shown in [Figure 4], the Beacon transmission probability of nodes not in the RSU reception range was higher than the Beacon transmission probability of nodes belonging to the RSU reception range. The RSU is a coordinator but continuously generates a beacon as a node, which increases the delay time of the beacon transmission. In addition, the beacon message transmission delay time is shorter when the inter-vehicle communication exchanges the beacon message with the nearby vehicle, compared to the vehicles communicating with the remote RSU.

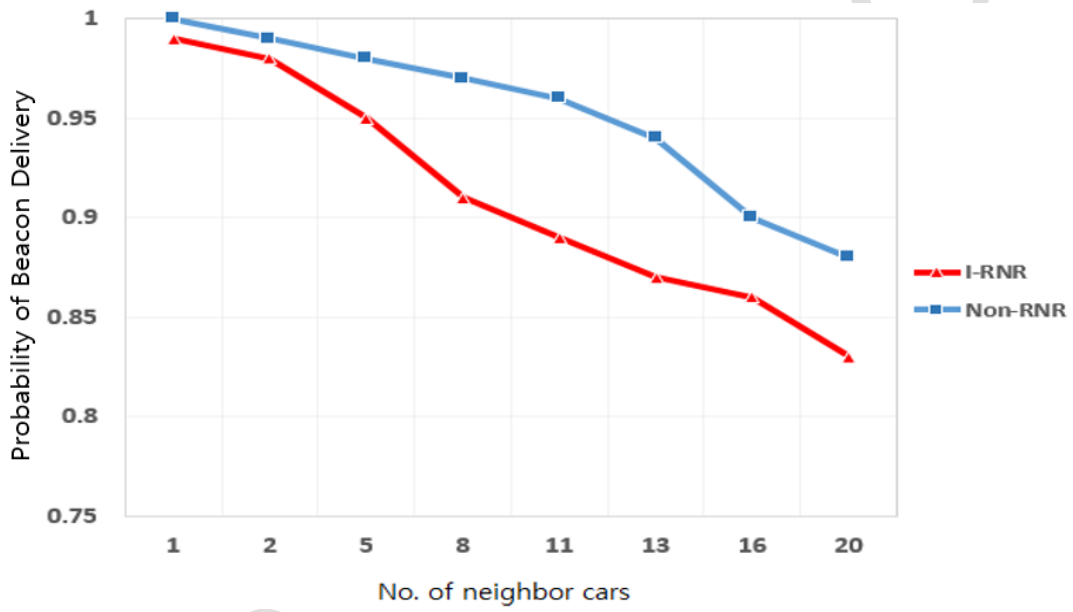

Figure 4. I-RNR and Non-RNR Beacon transmission probabilities

\section{Conclusion}

In this paper, we perform a virtual experiment using a network simulator before conducting a large-scale or actual test, and use the data obtained to predict the actual test result and present a method to increase the effectiveness of the experiment. Existing researches have also suggested ways to build a virtual experiment environment using network simulators. However, it is difficult to analyze the performance of each communication by analyzing the performance of all nodes without considering V2V and V2I communication. In this paper, we construct a road-like environment by combining the road traffic simulator SUMO, which supports a trafficlike environment in the real city, and the Veins framework for VANET wireless communication experiments. In this environment, we analyzed the performance of classification into V2I and $\mathrm{V} 2 \mathrm{~V}$ nodes according to RSU reception range, not performance analysis for all nodes. In this paper, it was confirmed that vehicles communicating outside the RSU receiving range have a higher probability of Beacon transmission than vehicles existing in the RSU receiving range. It is expected that it is suitable to communicate with $\mathrm{V} 2 \mathrm{~V}$ in a high vehicle density section and V2I in a low vehicle density section. 
Future research will analyze and experiment with the appropriate RSU placement distances for placing RSUs in high-density vehicles, such as intersections and highways, and where they are not. In addition, even if the RSU exists, the company plans to study how to improve throughput and reliability by switching to $\mathrm{V} 2 \mathrm{~V}$ communication according to vehicle density.

\section{Acknowledgements}

This research was supported by Basic Science Research Program through the National Research Foundation of Korea (NRF) funded by the Ministry of Education (No.2017R1D1A1B03032736).

\section{References}

[1] M.W. Ryu, S.H. Cha, and K.H. Cho, "An enhanced greedy message forwarding protocol for increasing reliability of mobile inter-vehicle communication," The Institute of Electronics Engineers of Korea, vol.47, no.4, pp.43-50, (2010)

[2] Uppoor S. and Fiore. M., "Large-scale urban vehicular mobility for networking research," Vehicular Networking conference (VNC), IEEE, pp.62-69, (2011) DOI: 10.1109/VNC.2011.6117125

[3] IEEE P1609.4, "Trial-use standard for wireless access in vehicular environments (wave) multi-channel operation," (2006) DOI: 10.1109/IEEESTD.2006.254109

[4] Veins, https://veins.car2x.org/documentation/

[5] Noori H., "Realistic urban traffic simulation as vehicular ad-hoc network(VANET) via veins framework," ISSN 2305-7254, (2012) DOI: 10.23919/FRUCT.2012.8122092

[6] A. Kopke, M. Swigulski, K. Wessel, D. Wilkomm, PT Haneveld, TEV Parker, OWVisser, HS Lichte, and S.Valentin, "Simulation wireless and mobile networks in OMNET++ the MiXiM vision," In Proceedings of the 1st international conference, pp.1-8., (2008) DOI: 10.4108/icst.simutools2008.3031

[7] OMNET++, https://omnetpp.org/

[8] MiXiM, http://mixim.sourceforge.net/

[9] Kit Guan Lim, Chun Hoe Lee, Renee Ka Yin Chin, Kiam Beng Yeo, and Kenneth Tze Kin Teo, "Simulators for vehicular ad hoc network (VANET) development," IEEE International Conference on Consumer Electronics-Asia (ICCE-Asia) pp.1-4, (2016) DOI: 10.1109/ICCE-Asia.2016.7804797

[10] Behrisch, M., Bieker, L., Erdmann, J., and Krajzewicz, D, "SUMO-simulation of urban mobility: an overview, SIMUL," The Third International Conference on Advances in System Simulation. ThinkMind. (2011)

[11] Krajzewicz D., Hertkorn G., Rössel C., and Wagner P., "SUMO (Simulation of Urban MObility) - an opensource traffic simulation," 4th middle East Symposium on Simulation and Modelling (MESM), pp.183-187, (2002) 